\title{
LA CONTAMINACIóN FLUVIAL Y LA ALTERACIóN DE USOS DEL SUELO Y PAISAJES COMO INDICADORES DE UN PROCESO DE URBANIZACIóN
}

\author{
Carlos A Vargas Cairo ${ }^{I}$
}

\begin{abstract}
RESUMEN
El acelerado proceso de urbanización y el modelo de desarrollo económico están causando un fuerte impacto en las zonas periféricas de Lima, como es el caso del distrito de Ate, el crecimiento de las actividades humanas en el distrito de Ate, ha generado el incremento de residuos en el Río Rímac, ocasionando la degradación de hábitat natural y la extinción de especies de la flora y fauna de la zona, los asentamientos humanos vierten los desagües al río, en forma directa, incrementando la contaminación de las aguas. El paisaje entorno al distrito de Ate, está fuertemente condicionado por estos procesos, la pérdida notable de suelos agrícolas han pasado a formar parte del suelo urbanizado y ahora la economía es un sector productivo básicamente de servicios.
\end{abstract}

Palabras clave: Urbanización, Contaminación, Paisaje.

\begin{abstract}
The accelerated process of urbanization and economic development model are having a serious impact on the outskirts of Lima, such as the district of Ate, the growth of human activities in the district of Ate has generated increased waste in the Rimac river, causing natural habitat degradation and extinction of species of flora and fauna, human settlements discharging wastewater into the river, directly, increasing water pollution. The landscape around the district of Ate, is strongly affected by these processes, remarkable loss of agricultural land have become part of the urbanized land and now the economy is basically a productive sector of services.
\end{abstract}

Keywords: urbanization, pollution, Landscape

\section{INTRODUCCIóN}

El campo de la Región de Lima Metropolitana recibe el impacto de un acelerado proceso de urbanización. Estas transformaciones territoriales, en el Perú y América Latina, se inscriben en el contexto mundial del nuevo orden económico, que se expresa a través de la globalización. Este proceso de crecimiento y expansión de la población de manera informal y no planificada en el ámbito de estudio, ha generado la contaminación del río Rimac y ha puesto en peligro espacios naturales y agrícolas que aún quedan en la cuenca media del río.
El río Rímac y los espacios agrícolas tuvieron una importancia económica y social para la población del distrito de Ate y alrededores; eran fuentes de abastecimiento de alimentos para el autoconsumo y comercialización, sin embargo, debido al proceso de expansión urbana, estos ecosistemas han ido degradándose y el área agrícola desapareciendo. Las características de este proceso se ven reflejadas en el crecimiento de las actividades humanas a lo largo del río Rímac; el incremento de residuos en sus aguas que ha ocasionado la degradación de hábitat natural y la extinción de especies de la flora y fauna de la zona, el cambio del uso del suelo y alteración del paisaje.

\footnotetext{
${ }^{1}$ Universidad Internacional de Andalucía-España. ecovar2002@yahoo.com
} 
Los asentamientos humanos en el distrito de Ate, no cuentan, en su gran mayoría, con un adecuado sistema de alcantarillado, vierten los desagües al río, en forma directa, incrementando la contaminación de las aguas.

Los cambios en el uso del suelo, la pérdida notable de suelos agrícolas que han pasado a formar parte de lo que hoy es suelo urbanizado y el paisaje en torno al distrito han sufrido una progresiva alteración y deterioro; la economía del distrito pasó de un sector primario de producción a un sector industrial y en la actualidad un sector productivo básicamente de servicios.

\section{MATERIAL Y MÉTODOS}

Para la presente investigación se utilizaron:

- Fotografías aéreas obtenidas del Instituto Geográfico Nacional:

. Fotografía aérea, escala 1/50,000 del 16 diciembre de 1961.

- Fotografía aérea, escala 1/30,000 de febrero y marzo de 1990.

- Fotografía aérea de Lima 2005- Internet: maps. google.com

- Planos del distrito de Ate (Municipalidad-Centro IDEAS).

- Mapa de las zonas criticas de la Cuenca del río Rímac. (Instituto Nacional de Defensa Civil)

- Fotografías panorámicas.

Cámara Pentax K-1000, lente Mitakon, objetivo $50 \mathrm{~mm}$, negativo Kodak de 400

ASA en formato $35 \mathrm{~mm}$.

Se trabajó teniendo como unidad ambiental el Distrito de Ate, en base a un análisis documental se sistematizó los datos sobre la calidad del agua del río Rimac en diferentes puntos; así mismo los cambios de usos del suelo y paisaje y se corroboró con fotografías aéreas, panorámicas y planos.

\section{RESULTADOS}

\section{MONITOREO DEL AGUA DEL RÍO Y LOS SIS- TEMAS DE ABASTECIMIENTO DE AGUA.}

1. La contaminación del agua del río, en el área de estudio, es el resultado del vertimiento de aguas servidas urbanas, desagües industriales y agrícolas, así como de residuos sólidos.

2. La disposición final de las aguas servidas sigue constituyendo un serio problema en el distrito la mayor parte de las aguas servidas, es decir el $52.56 \%$, se vierte al río y al suelo del entorno. La presencia máxima de materia orgánica en el río, ha registrado un aumento, en los últimos años (2000-2005).

3 Del 51.93\% de la población que usa el agua potable, la mitad corresponde a las urbanizaciones residenciales, a las que no tiene acceso la mayoría de la población de Ate; así mismo, el 52.4\% de la urbanizaciones populares (cooperativas de vivienda, asociaciones de vivienda y asociaciones pro-vivienda) cubre sus necesidades de agua y desagüe, el $47 \%$ a través de pilones o pozos y el $0.6 \%$ no tiene; en el caso de los asentamientos humanos marginales sólo el $2.4 \%$ posee conexiones domiciliarias, el $29.2 \%$ a través de pilones o pozos y el $68.4 \%$ carece de ellas.

4 Aproximadamente el $46 \%$ de las urbanizaciones populares (cooperativas de vivienda, asociaciones de vivienda y asociaciones pro-vivienda) del distrito carecen de infraestructura de desagüe; en los asentamientos humanos, el 100\%. Optan, entonces, por recurrir al uso de canales y acequias de regadío para eliminar las aguas servidas y los desechos sólidos.

PROCESO DE URBANIZACIÓN Y LOS CAMBIOS EN LOS USOS DEL SUELO Y PAISAJE

5 El proceso de urbanización en la ciudad de Lima y el distrito de Ate, se ha realizado sobre la base de la ocupación de tierras agrícolas y eriazas, y de manera desorganizada. Dicho proceso se ha caracterizado por un triple movimiento: del centro a la periferia, de la periferia al centro y de la periferia a otra periferia.

6 El distrito de Ate, en los últimos diez años ha duplicado el tamaño de su población, acentuando las características de ciudad intermedia, las estimaciones del año 2003 (INEI) alcanzan los 484,629 habitantes.

7 No existe un plan de ordenamiento territorial, lo cual favorece el uso inadecuado del suelo, ocupándose tierra agrícola para fines residenciales lo cual ejerce presión sobre la generación de residuos sólidos domésticos, convirtiendo las riberas del río Rímac en botaderos informales de basura.

8. El ámbito rural del distrito se situaba entre lo que son los poblados de Santa Anita, La Molina 
y Surco (hoy distritos), la zona de los alrededores de Puruchuco, Ceres y parte de los poblados de Vitarte, Santa Clara, Zona baja de Huaycán y Horacio Zeballos, en ambos márgenes del río Rimac, eran áreas destinadas al cultivo de flores, frutales, caña de azúcar, algodón, hortalizas y pan llevar.

9. En estos ámbitos, la superficie agrícola redujo en $72 \%$, entre 1972 y 1997, siendo Ate donde la pérdida del área agrícola fue del $90 \%$, así tenemos: 1986 (800 has), 1992 (571 has), 1996 (474.54 has), 2000 (289 has).

10. La invasión de tierras eriazas se debe a su carácter de bien público, son del estado. Estas tierras han sido ocupadas por la población para el desarrollo de un proceso precario de urbanización; que luego regularizan estableciendo asociaciones de vivienda para su reconocimiento.

11. El paisaje, en torno al distrito de Ate, está fuertemente condicionado por La presencia humana y los usos que el hombre hace del territorio: los paisajes naturales, las comunidades del río, ribera han sufrido una reducción y degradación de su estructura y pérdida de su diversidad biológica, algunos ámbitos han quedado confinados a espacios aislados, determinando una fragmentación de los habitats naturales.

12. Los paisajes culturales tradicionales, los ecosistemas que fueron manejados para la agricultura que proporcionan servicios ambientales y los ámbitos arqueológicos; han sufrido un progresivo deterioro, parte se ha perdido, otros se han transformado; conllevando también la perdida del conocimiento y cultura tradicional.

13. El gran número de migrantes que posee el distrito de Ate, hace difícil lograr una verdadera identidad con el distrito, por consiguiente, la poca valoración de sus recursos naturales, paisajes tradicionales y los monumentos arqueológicos que han quedado y que hoy están circunscritos en medio de la ciudad.

\section{REFERENCIA BIBLIOGRÁFICA}

ARDILES JARA, A. 1998. Los recursos naturales renovables -flora y fauna- y usos de la tierra en la Macrocuenca de Jabón Mayo, Canas, Cusco, Perú. Tesis de Maestría. Universidad Interna- cional de Andalucía. Sede Iberoamericana Santa María de La Rábida. Huelva-España.

BARRACÓ,H.,PARES,M.,PRAT,A.,TERRADAS ,J.1999. Barcelona 1985-1999. Ecologia d' una ciutat. Ajuntament de Barcelona-España.

BORJA, JORDI y MANUEL CASTELLS . 1997. Local y global. La gestión de las ciudades en la era de la información. Taurus. Madrid-España

CANO ORELLANA, A. 2002. Economía y sostenibilidad en las grandes aglomeraciones urbanas. Tesis doctoral. Sevilla-España.

CEPAL (Comisión Económica para América Latina y el Caribe). 1995. Población, equidad y transformación productiva. Santiago de Chile. Publicación de la Naciones Unidas.

COLLIER, DAVID. 1979. Barriadas y élites; de Odría a Velasco. Lima.

DE MATTOS, CARLOS. 2002. Redes, nodos y ciudades. Transformación de la Metrópoli Latinoamericana. Instituto de Estudios Urbanos y Territoriales. Facultad de Arquitectura, Diseño y Estudios Urbanos. Pontificia Universidad Católica de Chile.

DAMMERT EGO AGUIRRE, MANUEL. 2003. $L a$ descentralización en el Perú a inicios del siglo XXI: de la reforma institucional al desarrollo territorial.Volumen I, Instituto Latinoamericano y del Caribe de Planificación Económica y Social (ILPES), Santiago de Chile.

HUECK, K. 1978. Los bosques de Sudamérica. GTZ, Eschborn, 476 pp.

IBÁÑEZ BLANCAS, A. 2000. Bases de la gestión para la Microcuenca del Río Wankarmayo. Cusco. Perú. Tesis de Maestría. Universidad Internacional de Andalucía. Sede Iberoamericana Santa María de la Rabida. Huelva-España.

INSTITUTO NACIONAL DE ESTADÍSTICA E INFORMÁTICA. 2001. Perú: Estimaciones y proyecciones de población, 1950-2050. Urbana y Rural 1970- 2025. Boletín de Análisis Demográfico $\mathrm{N}^{\circ} 35$. Lima-Perú

KOEPCKE, H. W. 1961. Synokologische studien ander westseite der peruanischen Andes. Bonner geog. Abh., Heft 29, 320 pp. 
MATOS MAR, JOSÉ. 1977. Las barriadas de Lima. 1957. IEP. Lima-Perú

MURATORI, A. M.; BAENA, R.; GUERRERO, I.; HOLLMAN, V. 1996. Impacto ambiental en los ríos de Curitiba, Paraná-Brasil: entre la vitrina del Primer Mundo y la realidad del Tercer Mundo.

MINISTERIO DE AGRICULTURA, INRENA. 1996. Diagnóstico de la calidad del agua de la vertiente del Pacífico. Vol II-INR-43. Estudio de Contaminación y Preservación del río Rimac. Dirección de Protección del Medio Ambiente. Lima-Perú.

MUNICIPALIDAD DISTRITAL DE ATE. 2003. Boletines. Oficina de Planeamiento, Racionalización y Presupuesto. Municipalidad de Ate. Lima-Perú

NAREDO, J. M. y FRÍAS, JOSÉ. 2003. El metabolismo económico de la conurbación madrileña. 1984-2001. Revista Economía Industrial No 351. III. España.

OJEDA RIVERA, J. F. y GRANADOS CORONA, M. 1994. Paisaje y poblamiento. Edificaciones en el Parque Nacional de Doñana. Sevilla: Junta de Andalucía. Consejería de Obras Públicas y Transportes. España.
PANADERO MOYA, M. 2001. El proceso de urbanización de América Latina durante el periodo cientifico técnico. Revista bibliográfica de Geografía y Ciencias $\mathrm{N}^{\circ} 298$.

SANTOS, MILTON. 1996. De la totalidad al lugar. Oikos-Tauna. Barcelona. España.

SANTOS, MILTON. 1969. Mecanismos de crecimiento urbano en América Latina. Centro Latinoamericano de Pesquisas en Ciencias Sociales, 12,4, Río de Janeiro, 15 pp.

UNIVERSIDAD DE BARCELONA. 2001. El proceso de urbanización de América Latina durante el periodo científico-técnico. Revista Bibliográfica de Geografía y Ciencias Sociales, No 298 pp.

ZOIDO NARANJO, F. (Coordinador); CABALLERO SÁNCHEZ, J.; CARAVACA BARROSO, I.; FERNÁNDEZ SALINAS, V.; GIL ALONSO, M.; MADRID CALZADA, R.; MARCHENA GÓMEZ, M.; DEL MORAL ITUARTE, L.; OJEDA RIVERA, J.; OJEDA ZUJAR, J.; ROMERO VALIENTE, J.; SILVA PÉREZ, R. 2001. Informe de desarrollo territorial de Andalucía. Premio Andalucía 1999 de Economía y Hacienda Autonómica. Sevilla-España.

\section{ANEXOS}

Figura 1: Fotografía aérea del distrito de Ate 1961, que abarca hoy las 6 zonas del distrito la zona Ate-Mayorazgo y Vitarte Central; la zona de Santa Clara y lo que hoy es Huaycan y Horacio Zeballos, todas ellas eran extensas áreas agrícolas.

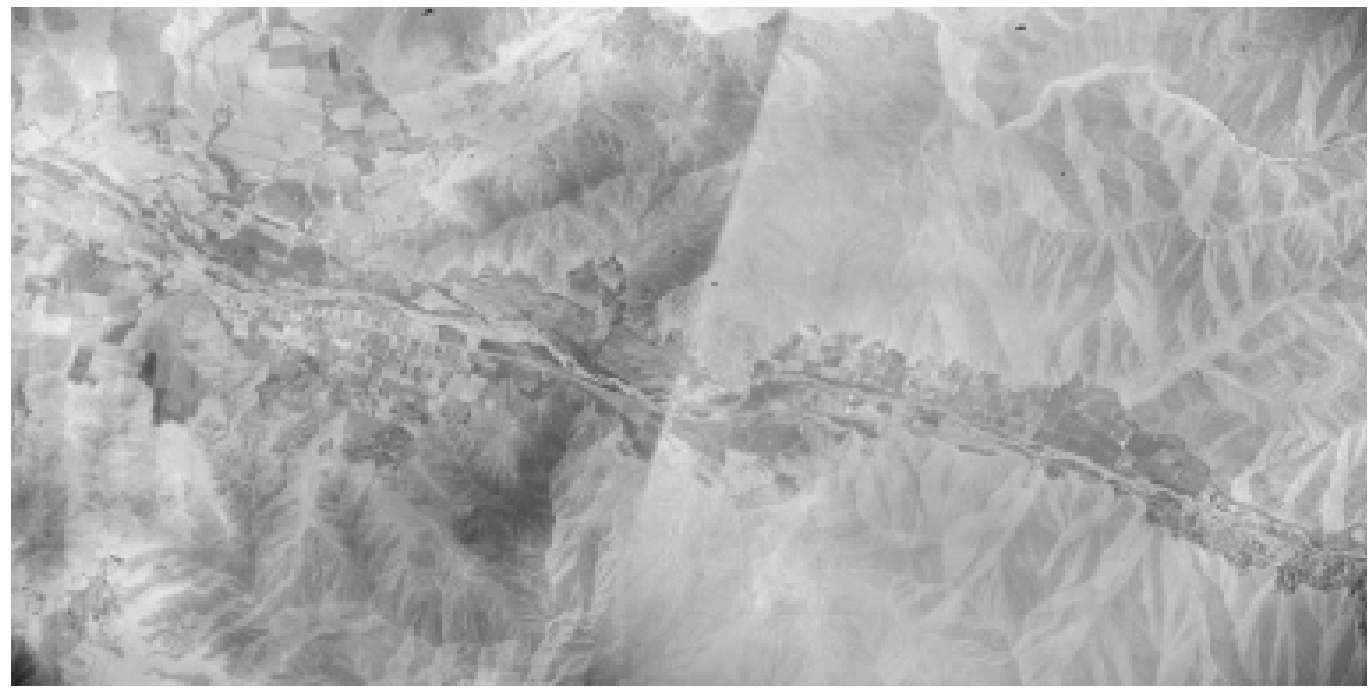


Figura 2: Fotografía aérea del distrito de Ate 1990, el centro de las zonas de Ate-Mayorazgo y Vitarte central; la zona Santa Clara y lo que es Huaycan y Horacio Zeballos, esta ultima zona no aparece en la fotografía anterior ya que se invadió en 1984. Las áreas agrícolas fueron sustituidas por urbanizaciones e invasiones.

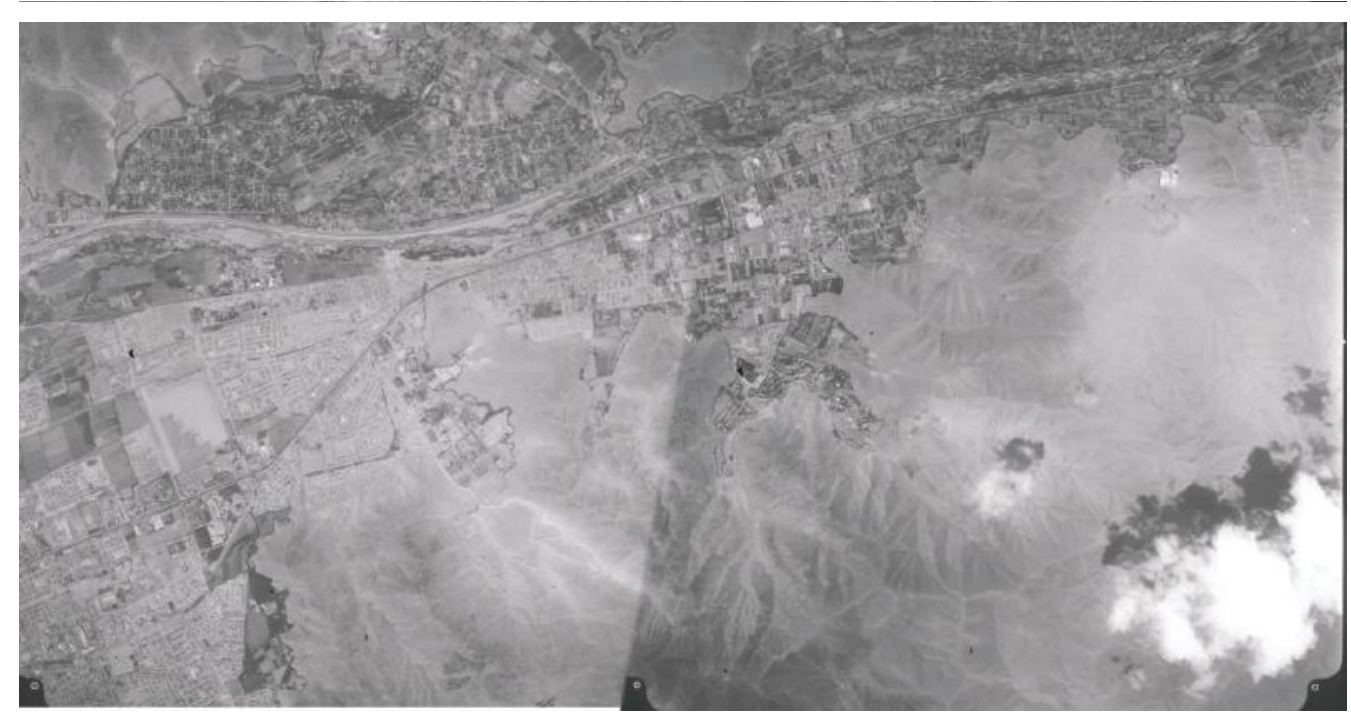

Fitura 3: Procesos informales de urbanización y suelo agrícola urbanizado
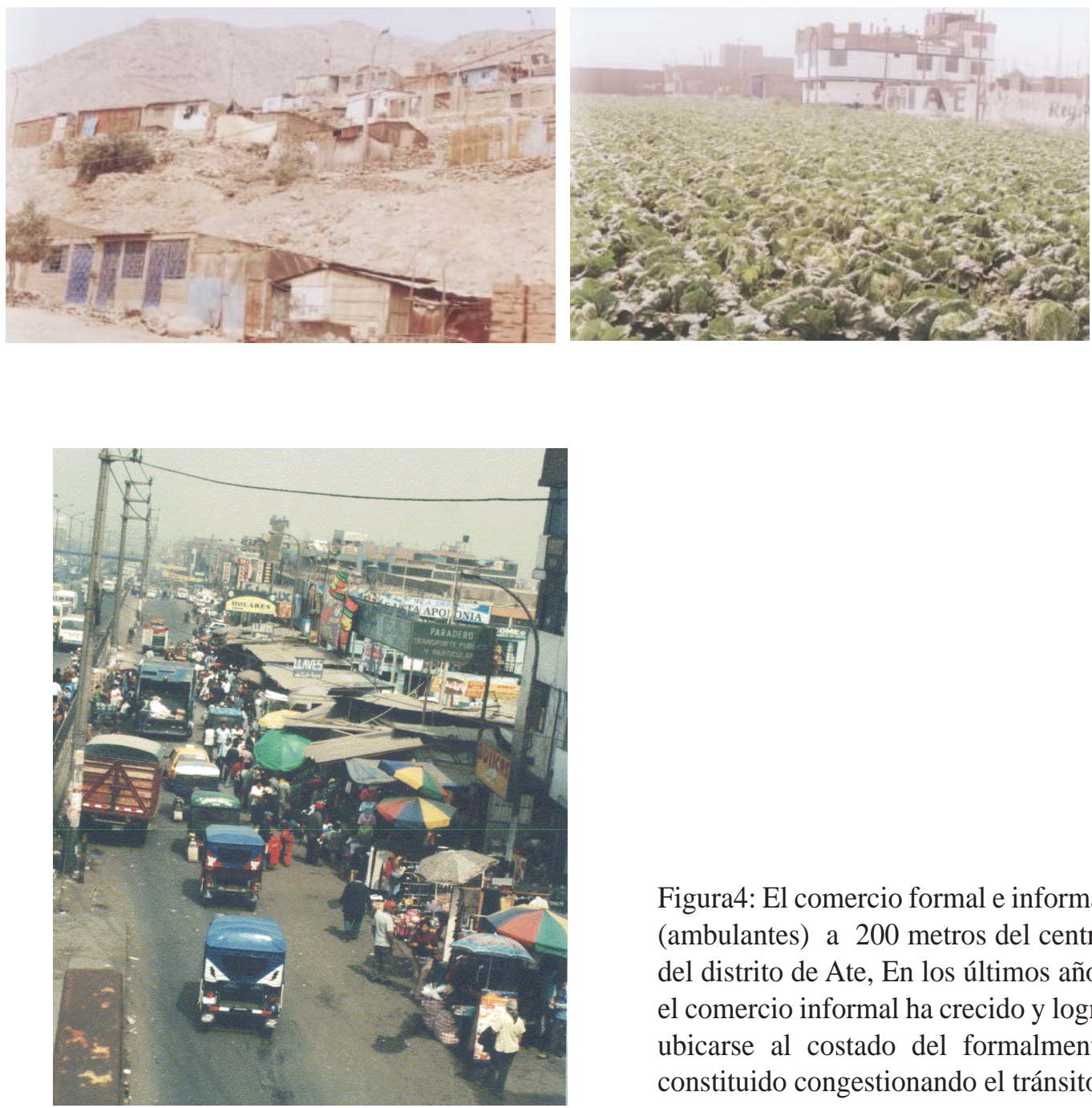

Figura4: El comercio formal e informal (ambulantes) a 200 metros del centro del distrito de Ate, En los últimos años el comercio informal ha crecido y logra ubicarse al costado del formalmente constituido congestionando el tránsito. 
Figura 5: Estas dos fotografias aéreas de 1961 muestra el poblado de Vitarte y sus espacios agrícolas.
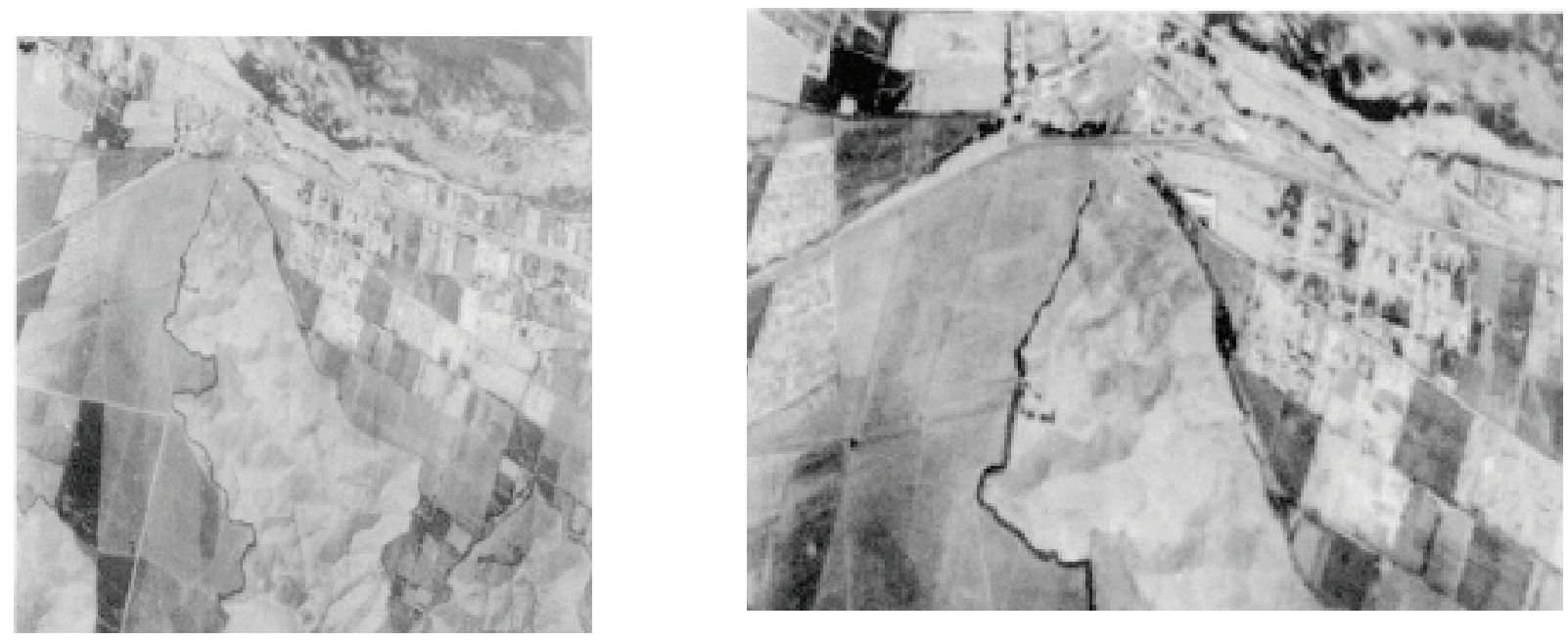

Figura 6: Los pobladores de las invasiones denominadas Asentamientos Humanos AA.HH. van desplazándose y ocupando espacios y construyendo sus viviendas, en esta fotografía se muestra el Local Comunal del AA. HH. Amauta II- Ate-1995

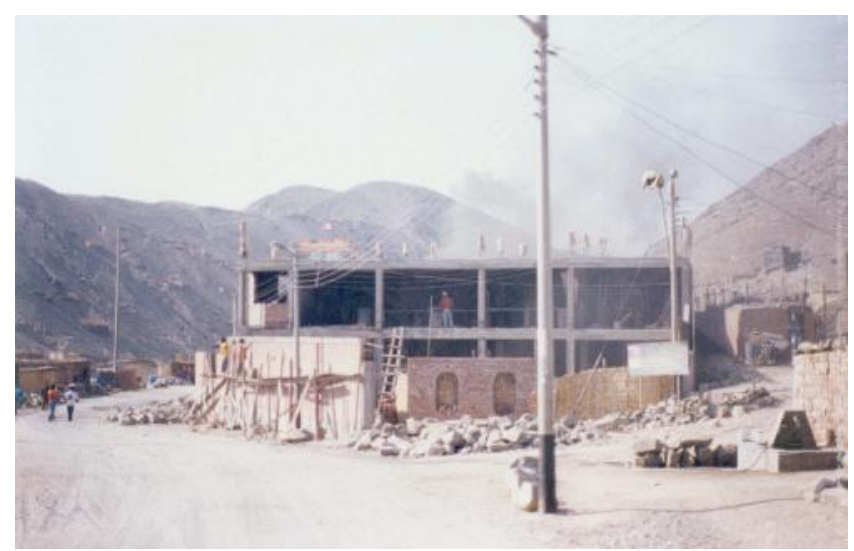

Figura 7: En esta fotografía podemos observar el mismo Local Comunal después de años (2005) y claramente se observa el crecimiento poblacional mayor número de invasores y el servicio de transporte público aumenta, así como la presión por servicios básicos.

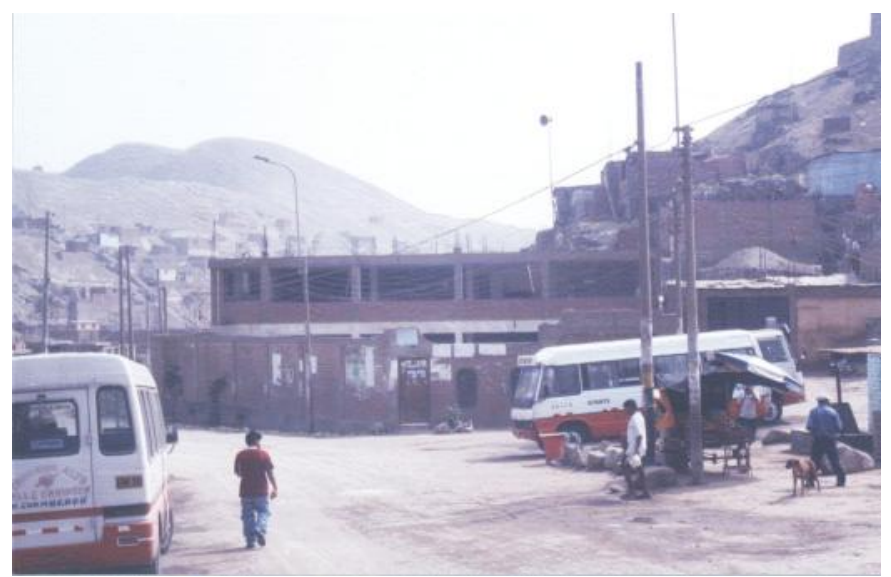

